\title{
antibodies
}

ISSN 2073-4468

www.mdpi.com/journal/antibodies

Review

\section{The PARP1/ARTD1-Mediated Poly-ADP-Ribosylation and DNA Damage Repair in B Cell Diversification}

\author{
Jackline J.M. Lasola ${ }^{1, \dagger}$, Andrea Hodgson ${ }^{1,2, \dagger}$, Xin Sun ${ }^{1, \dagger}$ and Fengyi Wan ${ }^{1,3,4, *}$ \\ 1 Department of Biochemistry and Molecular Biology, Bloomberg School of Public Health, \\ Johns Hopkins University, Baltimore, MD 21205, USA \\ 2 Department of Molecular Microbiology and Immunology, Bloomberg School of Public Health, \\ Johns Hopkins University, Baltimore, MD 21205, USA \\ 3 Department of Oncology, School of Medicine, Johns Hopkins University, Baltimore, MD 21205, USA \\ 4 Sidney Kimmel Comprehensive Cancer Center, Johns Hopkins University, Baltimore, MD 21287, USA \\ $\dagger$ These authors contributed equally to this work. \\ * Author to whom correspondence should be addressed; E-Mail: fwan@jhsph.edu; \\ Tel.: +1-410-955-5848; Fax: +1-410-955-2926.
}

Received: 10 October 2013; in revised form: 6 January 2014 / Accepted: 10 January 2014 /

Published: 16 January 2014

\begin{abstract}
ADP-ribosylation is an essential post-translational modification, mediated by a family of proteins named poly-ADP-ribose polymerases/Diphtheria toxin-like ADPribosyltransferases (PARPs/ARTDs), that functions to assist in cellular homeostasis through an array of mechanisms. Although the function of PARP1/ARTD1-mediated polyADP-ribosylation (PARylation) in response to environmental genotoxic stressors has been extensively studied, its role in the regulation and maintenance of cellular events under times of programmed DNA damage and repair remains to be elucidated. In the case of B cell maturation and differentiation, processes such as $\mathrm{V}(\mathrm{D}) \mathrm{J}$ recombination, somatic hypermutation, and class switch recombination, require the induction of DNA strand breaks for the generation of a varied immunoglobulin repertoire and, thus, serve as a model system to explore the function of PARylation in immunological processes. In this review, we summarize the current understanding of ADP-ribosylation and the PARPs/ARTDs family proteins, in particular PARP1/ARTD1-conferred PARylation, in B cells. Following an overview of PARylation in cellular responses to environmental and spontaneous DNA damage, we discuss the emerging function of PARP1/ARTD1 and PARylation in DNA damage-induced nuclear factor kappaB (NF- $\mathrm{B})$ signaling and $\mathrm{B}$ cell maturation and
\end{abstract}


differentiation. Finally, we conclude by underlining further efforts that are needed to understand how the PARPs/ARTDs family proteins and ADP-ribosylation control the development and function of B cells.

Keywords: PARP1/ARTD1; Poly-ADP-ribosylation; PAR; DNA damage; NF-кB; B cells

\section{Introduction}

Post-translational modifications (PTMs) help maintain homeostasis of cells by regulating protein turnover, subcellular localization, and interaction affinity [1]. Many of these modifications result from cellular responses to environmental cues that require rapid cellular adaption to challenges [1]. In the case of immune cells, such as B lymphocytes, environmental stimuli serve as one of the most important drivers of cellular differentiation and proliferation, ultimately generating a complete immunoglobulin repertoire.

ADP-ribosylation is a unique PTM, which includes mono-ADP-ribosylation (MARylation) and poly-ADP-ribosylation (PARylation), that add one or more (up to a few hundred) negatively charged ADP-ribose moieties from nicotinamide adenine dinucleotide (NAD) to target proteins [2]. In response to both intrinsic and extrinsic signals, poly-ADP-ribose polymerases/Diphtheria toxin-like ADPribosyltransferases (PARPs/ARTDs) regulate the majority of ADP-ribosylation events in cells, and in the process, control a wide array of cellular processes including, but not limited to, DNA repair, chromatin structure remodeling, transcriptional regulation, cell death, cell division, and cell differentiation $[2,3]$. In the last decade, emerging evidence has highlighted the functional role of ADPribosylation and ADP-ribosylating enzymes in innate and adaptive immune responses [4]. It is well accepted that the development of an effective acquired immune system relies on the ability to generate a diverse $\mathrm{B}$ and $\mathrm{T}$ lymphocyte pool capable of responding to the immense variety of antigens a host may encounter. A hallmark of the acquired immune system is the ability to adapt to the invading pathogen, so that upon seeing a novel antigen, circulating $\mathrm{B}$ and $\mathrm{T}$ lymphocytes specific to that antigen can become activated and proliferate. For B cells in particular, part of this proliferation will include fine tuning the specificity of the receptor via class switching and somatic hypermutation, discussed in more detail later in this review, which require self induced DNA damage. PARPs/ARTDs have been revealed to be critical for lymphocyte development, activation, proliferation, and differentiation [3] and their role in DNA damage recognition and repair indicate a link worthy of study. Here we intend to contextualize the current understanding of functional characteristics of ADP-ribosylation, especially PARP1/ARTD1-mediated PARylation in B lymphocytes and provide future directions for expanding the knowledge of this essential PTM in B cell development and activity.

\section{PARylation is Essential for Cellular Responses to Environmental and Spontaneous Stresses}

\subsection{PARylation Homeostasis: PARPs/ARTDs, PARG, and Others}

The PARPs/ARTDs family of proteins (17 known in humans and 16 in mice) shares a highly conserved sequence signature $[5,6]$. In contrast to most PARPs/ARTDs that confer MARylation, some 
PARPs/ARTDs (i.e., PARP1/ARTD1, PARP2/ARTD2, PARP5A/ARTD5, and PARP5B/ARTD6) are known to catalyze PARylation [7]. As the founding member of this superfamily, PARP1/ARTD1 has been extensively studied and remains the best characterized of the PARPs/ARTDs superfamily thus far. Mounting evidence from genetic (in particular three independent PARP1/ARTD1-deficient mouse models), pharmacological, and epidemiological studies underscores the function of PARP1/ARTD1 as a critical survival factor that plays essential roles in DNA damage responses $[2,3,8-10]$. The enzymatic structure of PARP1/ARTD1 is widely believed to consist of four distinct domains: (1) an N-terminal DNA binding domain, serving as a DNA damage sensor; (2) a nuclear localization signal (NLS), harboring a caspase-3 cleavage site for downregulating PARP1/ARTD1 function; (3) an automodification domain, possessing a BRCT domain for partner binding and PARP1/ARTD1 release; and (4) a C-terminal PARP domain for catalytic activity [11]. It is noteworthy that in the presence of DNA lesions, the DNA-binding domain of PARP1/ARTD1 binds damaged DNA and such recognition induces conformational changes to PARP1/ARTD1 and elevates its catalytic activity by almost 500-fold [2]. With such enhanced catalytic activity, PARP1/ARTD1 robustly synthesizes and supplements polymers of ADP-ribose to target proteins, such as PARP1/ARTD1 itself, histones, DNA repair proteins, transcription factors, and chromatin regulators $[4,12,13]$. It is therefore not surprising that PARP1/ARTD1- and other PARPs/ARTDs-catalyzed PARylation orchestrates various signaling cascades leading to a wide range of cellular processes. Beyond its well-known DNA repair function, PARylation has also been revealed to modulate the transcriptional activity of several transcription factors and fine-tune their gene transcription in response to the extracellular signal-regulated kinases (ERK) signaling pathway [14-18]. Moreover, the PARP1/ARTD1-regulated PARylation that occurs in response to genotoxic stress in the nervous system has also been suggested to play a key role in the development of neurodegenerative diseases such as Parkinson's disease [19-21].

A class(es) of enzymes that can reverse the actions of PARPs/ARTDs is required to lower the levels of ADP-ribosylation, especially PARylation, in order to maintain tight regulation of PAR homeostasis [3,22-24]. Poly-ADP-ribose glycohydrolase (PARG), ADP-ribosyl hydrolase (ARH), and nucleoside diphosphate linked to another moiety X (NUDIX) family of proteins are very important enzymes to remove, degrade, and recycle PAR chains $[3,24]$. In particular, PARG possesses both exoor endo-glycosidase activities and cleaves the glycosidic bonds between the ADP-ribose units, thus quickly hydrolyzing most of ADP-ribose polymers on PARylated proteins [25]. That said, the macrodomain-containing proteins (MDCPs), such as MacroD1, MacroD2, and C6orf130, were recently revealed to be the enzymes that fully reverse the affects of PARPs/ARTDs by hydrolyzing the terminal ADP moiety [22,23]. The significance of controlled degradation of PAR chains under pathophysiological settings, in particular during the DNA damage response, is highlighted by recent studies with micro-irradiation showing efficient recruitment of PARG isoforms and MacroD2 to DNA damage foci $[23,26]$. In support of this notion, deletion of the nuclear PARG isoform in embryonic stem cells and mice significantly increases their sensitivity to alkylating agents and $\gamma$-irradiation [27-30]. Taken together, PARPs/ARTDs, PARG, MDCPs, as well as other PAR modifying enzymes harmonize to maintain tight control of the metabolic turnover and recycling of PAR, hence achieving rapid and efficient regulation of multiple cellular processes in a spatially and temporally controlled manner (Figure 1). 
Figure 1. Homeostasis of ADP-ribosylation. ADP-ribosylation is up-regulated by polyADP-ribose polymerases/Diphtheria toxin-like ADP-ribosyltransferases (PARPs/ARTDs) and ADP-ribose transferases (ARTs), adding one or more ADP-ribose from NAD to target proteins, and down-regulated by another group of enzymes poly-ADP-ribose glycohydrolase (PARG), ADP-ribosyl hydrolase (ARH), nucleoside diphosphate linked to another moiety X (NUDIX), and macrodomain-containing proteins (MDCPs), degrading and removing poly-ADP-ribose (PAR) or mono-ADP-ribose (MAR) chains. The ADPribosylation modifying enzymes function jointly to coordinate the metabolic turnover and recycling of ADP-ribosylation.

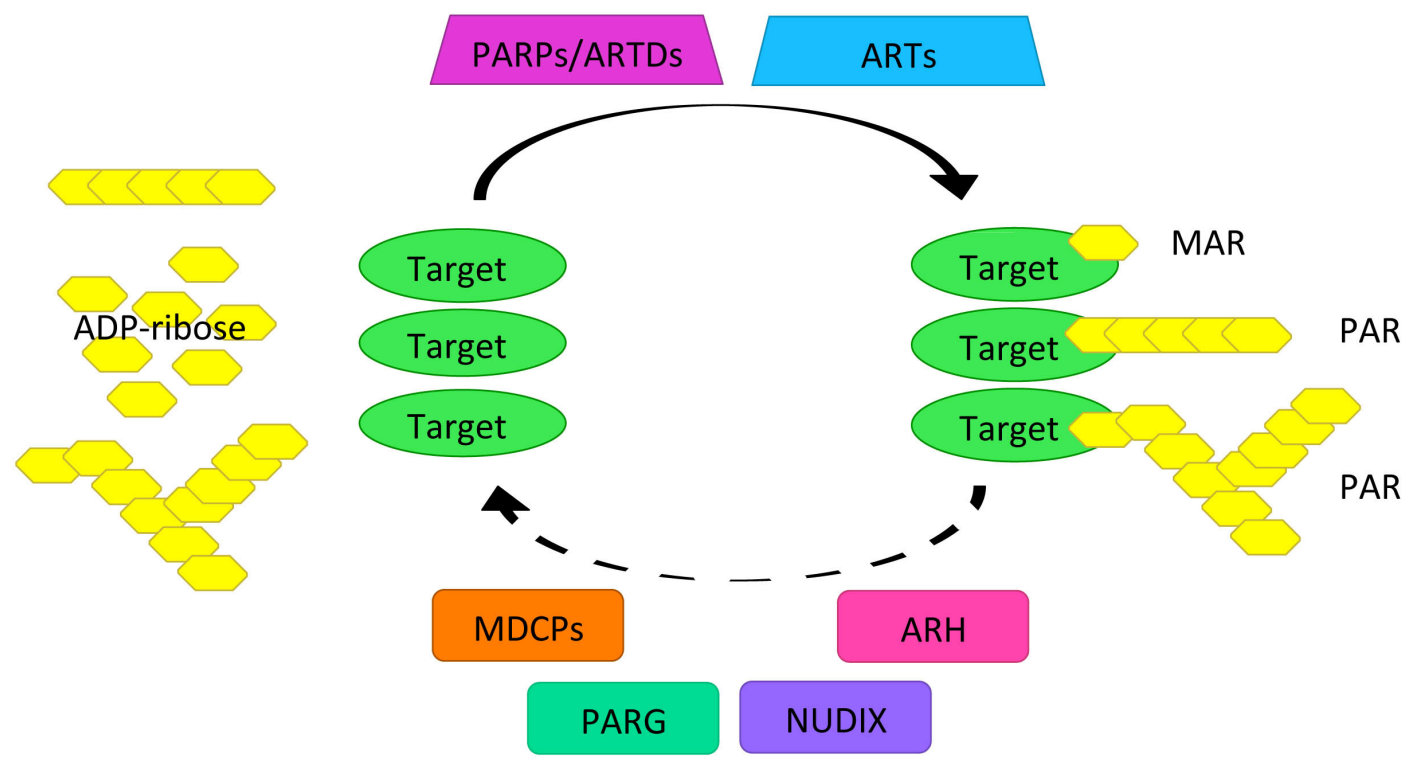

2.2. Role of PARylation as of a Platform for the DNA Damage Repair Protein Complex Assembly and Maintenance of Genomic Stability

The formation of distinct foci in the nuclei of cells suffering genotoxic stress is believed to be one of the hallmarks of PAR synthesis in response to DNA damage. The relevance of such an amplified PAR signal is believed to label the damaged sites and to indicate the incidence as well as the magnitude of DNA damage [2]. More importantly, as demonstrated by numerous previous studies, focal PARylation at DNA damage lesions also functions to create the appropriate chromatin microenvironment to facilitate access for the repair machinery and to serve as a docking platform for the focal assembly of the DNA repair complex, hence, orchestrating the appropriate DNA damage response signaling cascades $[31,32]$.

Various lysine residues at the amino-terminal tail of the core histones were identified to be linked with PAR chains covalently [32], and such PARylation is believed to favor loosening chromatin structure around DNA damage sites [32-34]. Moreover, earlier studies suggest that PARP1/ARTD1catalyzed PAR synthesis could be another mechanism for targeting chromatin-remodeling complexes to damaged chromatin by promoting the accessibility of checkpoint and repair factors. Macrodomaincontaining chromatin remodeling enzyme Amplified in Liver Cancer 1 (ALC1, also known as CHD1L) was shown to be rapidly recruited to DNA damage sites and to activate its nucleosome- 
repositioning function in a PAR-dependent manner [35,36]. Conversely, recent findings outlined a role for PARP1/ARTD1 in transcriptional silencing by triggering chromatin compaction, which can be best exemplified by the finding that several components of the NuRD complex, including chromodomain helicase DNA binding protein 4 (CHD4) are recruited by PARP1/ARTD1 and execute histone deacteylase and demethylase activities [37,38]. Although there is controversy surrounding its chromatin functions, PARP1/ARTD1-catalyzed PARylation has been proposed as an important PTM to regulate chromatin microenvironment.

Equally important, a novel function has been described where PAR chains function as a docking platform for the DNA repair complex assembly. In support of this notion, previous in vitro studies demonstrate that PAR chains have direct or indirect interactions with an increasing list of DNA repair proteins involved in almost all known DNA repair pathways, yet future studies are still needed to confirm these interactions in vivo. For instance, the scaffold protein XRCC1 (X-ray repair crosscomplementing protein 1), a member of the single-strand break repair (SSBR) and base excision repair (BER) pathways, exhibits a higher affinity to PARylated PARP1/ARTD1 in comparison to regular PARP1/ARTD1 [39]. Importantly, the recruitment of XRCC1 to damaged DNA lesions is dramatically attenuated in the absence of catalytically active PARP1/ARTD1 [40]. Moreover, earlier studies reported that DNA-dependent protein kinase (DNA-PK), an essential kinase in the non-homologous end joining (NHEJ) pathway, and PARP1/ARTD1 have both physical and functional interactions [41], and that the activation of ataxia telangiectasia, mutated (ATM) in the homologous recombination (HR) pathway, is significantly reduced in the presence of PARP1/ARTD1 inhibitors [42]. PARylation has therefore emerged as an additional key PTM to orchestrate DNA damage repair signaling in the nucleus.

\subsection{PARylation in Cellular Responses to Programmed DNA Damage}

It is well known that somatic recombination in spermatogenesis, retroviral integration, and $\mathrm{T}$ and $\mathrm{B}$ lymphocyte development are processes that require programmed DNA double-strand breaks (DSBs) and that many DNA repair proteins involved in repairing exogenous DNA damage also play a major role in repairing programmed DNA damage [43]. These overlapping functions are underscored when defects in DNA repair proteins are associated with sterility disorders, neurodegeneration, cancer, and immunodeficiency. For instance, PARP1/ARTD1 and PARP2/ARTD2 knockout mice harbor defects in germ cell development $[44,45]$.

Germ cells undergo programmed DSBs during meiosis and increase genetic diversity via recombination and crossover events between homologous chromosomes, which require efficient repair for proper chromosomal segregation. Studies in primary rat spermatocytes have detected PARP1/ARTD1 and PARP2/ARTD2 catalyzed PAR synthesis [44] activated by the protein-bound DSBs generated by topoisomerase II beta (topoisomerase II $\beta$ ) in order to remove DNA supercoiling [46]. These PAR chains facilitate loosening chromatin DNA by removing the core histone variants and histone linker proteins [46]. In PARP1/ARTD1- and PARP2/ARTD2-deficient mice, reduced PARylation leads to the persistence of unrepaired DSBs during spermatogenesis, which result in the accumulation of DNA lesions in mature spermatozoa, male infertility, and defective embryonic development $[44,45]$. Similarly, PARP1/ARTD1, as shown in a recent study [47], was revealed to be indispensible in maintaining chromosome integrity during meiosis in female germ cells. Of note, deletion of the 
nuclear PARG $110 \mathrm{kDa}$ isoform in mice results in aberrant PARylation and these nuclear PARG knockout mice display subfertility due to abnormal sperm nuclei shape and DNA strand break accumulation in sperm [48]. Thus, PAR metabolism is being appreciated more as a key regulator during germ cell development [44].

The integration of latent viral genomes into infected host cells is another avenue in which PARylation has been linked to programmed DNA damage, due to the need to repair and ligate the host DNA with the invading viral DNA. This is particularly important for retroviral infections where integration is a key step in the viral life cycle [49]. Viral integrase enzymes induce DSBs in both viral and target cellular DNA strands, leaving a four- to six-base gap and a two-base mismatch that have to be repaired. The mismatch repair network and the DNA damage sensors ATM and DNA-PK have been described to facilitate viral genome integration [50]. Inhibition of PARP1/ARTD1 by chemical inhibitors, antisense oligonucleotides, as well as dominant-negative blockers, has been shown to remarkably decrease the infectivity of mammalian cells by recombinant retroviral vectors $[51,52]$. Moreover, PARP1/ARTD1 has been reported to promote the integration of the human immunodeficiency virus (HIV) genome near the centromere region [53], and deletion of PARP1/ARTD1 in mouse fibroblasts eliminated pseudotyped HIV type I infection by blocking the integration step [54]. It is noteworthy that other studies show that alteration in various DNA repair sensors has no effect on HIV integration [55-57], therefore more studies are needed to conclusively place DNA repair pathways as an integral part of the provirus formation.

\section{Emerging Role of PARylation in B Cell Function}

\subsection{DNA Damage, $N F-\kappa B$, and B Cell Development}

Similar to germ cell development and viral genome integration into host cells, programmed DNA damage and repair is also an important process in lymphocyte development [58]. In order to create a broad and varied antibody repertoire, B lymphocytes undergo somatic mutations and recombination activating gene (RAG) dependent $\mathrm{V}(\mathrm{D}) \mathrm{J}$ recombinations, making them an interesting model for the study of DNA damage responses in general, and for the purposes of this review, the involvement of PARPs/ARTDs and PARylation. These rearrangements allow for the generation of B cells that can produce related antibodies using similar variable regions, yet through class switching and affinity maturation these antibodies have diverse functions and varying degrees of specificity $[59,60]$. DNA lesions generated during lymphoid development induce cellular responses that activate the cell-cycle checkpoint and initiate DNA repair. Previous studies from mice deficient in DNA damage-responding or damage-repairing factors and human patients with mutations in those genes have provided insight into our understanding of the DNA damage response, cell-cycle checkpoint, and DNA repair in B cell development, differentiation, and maturation [60].

Nuclear factor kappa B (NF- $\kappa \mathrm{B})$, originally identified as a mechanism governing B lymphocyte-specific transcription of immunoglobulin (Ig) kappa ( $\kappa$ ) light chain gene [61], is one of the most important transcription factors given its involvement in almost all steps of $\mathrm{B}$ cell development $[62,63]$. Based on sequence homology, the mammalian NF-kB consists of five Rel family proteins, i.e., RelA (p65), RelB, c-Rel, p50, and p52 [64,65]. Recent studies suggest that other proteins beyond Rel subunits, 
such as ribosomal protein S3 (RPS3) [66] and Src-associated substrate during mitosis of $68 \mathrm{kDa}$

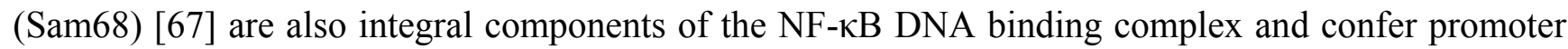
selectivity and transcriptional specificity of NF-kB $[68,69]$. During the last two decades, a great number of findings from biochemical, molecular, and genetic studies have illustrated the essential role of NF- $\kappa \mathrm{B}$ in $\mathrm{B}$ cell development and function [62,63]. NF- $\kappa \mathrm{B}$ functions in immature $\mathrm{B}$ cell development in the bone marrow [70-72], peripheral B cell differentiation [63,73], mature $B$ cell survival [74], and mature B cell response to various stimuli [75]. Notably, a recent study demonstrated that the synergistic interaction between RPS3 and p65 within the NF- $\mathrm{BB}$ complex is required for B cell receptor editing [72]. More importantly, previous studies on human patients with mutations in the gene encoding I $\mathrm{B} \mathrm{B} \alpha$ kinase gamma (IKK $\gamma$ ), a central molecule in the DNA damage-activated NF- $\mathrm{B}$ signaling pathway [43], suggest that the mutations on the C-terminal ZF domain of IKK $\gamma$ specifically cause defects in the DNA damage NF- $\kappa$ B pathway without affecting lipopolysaccharide (LPS)stimulated canonical NF- $\kappa \mathrm{B}$ activation [76,77]. Hence, these studies provide the crosstalk between spontaneous DNA damage/repair and intrinsic NF- $\kappa \mathrm{B}$ activity in $\mathrm{B}$ cells and underscore the specific requirement of DNA damage dependent NF- $\mathrm{B}$ activation for $\mathrm{B}$ cell function.

\subsection{The Critical Role of PARylation in DNA Damage-Induced NF- $\kappa B$ Activation}

Previous studies have demonstrated that PARP1/ARTD1 is essential for DNA damage-induced NF- $\kappa B$ transcriptional activity, albeit with various proposed mechanisms [78-81]. Earlier works showed that PARP1/ARTD1 was apparently not involved in the signaling that leads to inhibition of NF- $\kappa \mathrm{B}$ alpha $(\mathrm{I} \kappa \mathrm{B} \alpha)$ degradation and p65 nuclear translocation following DNA damage [79,81]. Rather, PARP1/ARTD1 was proposed to regulate the transcriptional activity of NF- $\kappa \mathrm{B}$ p65 subunit at the chromatin level through an interaction with the N-terminal Rel homology domain (RHD) of p65 [79,81]. In contrast to these findings, a recent study by Scheidereit and colleagues demonstrates that PARP1/ARTD1 and PARylation are required to bridge the cytoplasmic activation of the IKK complex and the nuclear sensing of DNA damage [78]. PARP1/ARTD1 and PARylation have been revealed to be necessary and required for rapid assembly of a signaling complex comprised of IKK $\gamma$, protein inhibitor of activated STAT Y (PIASy), and ATM following DNA damage, which results in the post-translational modification of IKK $\gamma$ by the small ubiquitin-related modifiers (SUMO), an essential step for DNA damage-induced NF- $\kappa B$ activation [78]. It is notable that the formation of this signalosome involves the catalytic activity and DNA binding domain of PARP1/ARTD1 as well as the PAR-binding domain present in the C-terminus of PIASy [78,82]. In line with these findings, previous in vitro studies using recombinant proteins suggest that PAR chains enhance the binding affinities of IKK $\gamma$, PARP1/ARTD1 and PIASy [78]. Moreover, PAR synthesis has been illustrated to be required for IKK $\gamma$-PARP1/ARTD1-PIASy interactions of cells in vivo $[78,82]$. In parallel to DNA repair and cell cycle arrest, DNA damage-induced NF- $\kappa B$ activation is well known to lead to either cell survival signals or cell death signals. If PARP1/ARTD1 and PARylation were placed prior to these two key branches of the signaling cascades initiated from DNA damage lesions, it would suggest that the dynamic regulation of PAR chains, as well as other PTMs such as SUMOylation and phosphorylation, at the DNA damage foci can elegantly provide specificity for the interactions of numerous signaling proteins and momentously influence the cellular decisions for survival or death. 


\subsection{Expanding Role of PARylation in B Cell Functions}

$\mathrm{V}(\mathrm{D}) \mathrm{J}$ recombination is the process whereby immunoglobulin $(\mathrm{Ig})$ variable regions are created by joining individual variable $(V)$, diversity $(D)$, and joining $(J)$ gene segments that are encoded along the chromosome. This creates immense diversity in the antibody repertoire and requires the recombination activating genes 1 and 2 (Rag1 and Rag2) to recognize and cleave the DNA at specific sequences, and the DNA repair machinery of the NHEJ pathway to connect the subsequent gene segments $[83,84]$. Since lymphocytes undergo V(D)J recombination to generate their cell receptors, B cells and T cells fail to develop properly when there are mutations in NHEJ pathway proteins and the programmed DNA damage cannot be efficiently repaired [83]. While PARP1/ARTD1-, PARP2/ARTD2-, and PARP3/ARTD3-deficient mice develop mature splenic B and T cells, indicating that these proteins are not required for receptor assembly [85-87], they do exhibit abnormal levels of basal immunoglobulins. Moreover, minor defects in T cell receptors [88] as well as and higher levels of $\gamma \mathrm{H} 2 \mathrm{AX}$ accumulated in thymocytes [89] have been reported in PARP2/ARTD2-deficient mice. Yelamos and colleagues concluded that these differences could be attributed to increased apoptosis of T cells undergoing TCR rearrangement because of inadequate resolution of DNA damage [90]. These evidences suggest that PARP2/ARTD2 catalytic activity may be required to compensate for the cost of generating DSBs by RAG-1/RAG-2 during V(D)J recombination in T cells.

Birds and other farm animals utilize immunoglobulin gene conversion to increase the diversity of their variable regions of the B cell receptor by transferring sequences between rearranged $V$ regions and $V$ region pseudo-genes located upstream on both the $\operatorname{IgH}$ and $\operatorname{IgL}$ loci [91,92]. Studies employing DT40 cells, a chicken B cell line model for Ig gene conversion, have demonstrated that PARP/ARTD inactivation or disruption of its BRCT domain hampers Ig gene conversion and increases accurate repair [93,94], while these studies are compelling, these chicken cells do not express all the PARP/ARTD proteins and therefore their implications could be limited and future studies will be needed to demonstrate these principles in human cell lines. One hypothesis suggesting that PARP1/ARTD1 inhibits Ku70-mediated recruitment of DNA ligase IV (where Ku70 is an integral protein of the NHEJ pathway) stems from studies showing a direct interaction of PARP1/ARTD1 BRCT domain and Ku70, Ku80, and DNA-PK. In fact, PARP1/ARTD1 was shown to compete with Ku-70 for binding to the DNA lesion and promote alternative NHEJ in contrast to the Ku-70/DNA ligase IV induced traditional NHEJ [93,95]. In support of this notion, this study also determined that inactivation of PARP1/ARTD1 and Ku70 or PARP1/ARTD1 and DNA ligase IV rescues Ig gene conversion [93] and other work has shown that deficiency of $\mathrm{Ku} 70$ predisposes the cell toward PARP1/ARTD1-dependent end-joining [96]. This alternative NHEJ is more mutagenic than classical NHEJ due to minor deletions at the ends of the lesion to facilitate repair [97]. One could speculate that a PARP1/ARTD1 directed preference for alternative NHEJ could be advantageous to the host as increased mutagenesis could help improve receptor diversity providing another mechanism facilitating a varied $\mathrm{B}$ cell repertoire and underlining the importance of PARP1/ARTD1 mediated DNA damage repair.

Somatic hypermutation is another mechanism of increasing antibody affinity more commonly occurring in humans than gene conversion. It arises when the activation-induced cytidine deaminase (AID) enzyme deaminates cytosines, changing them to uracil residues that are recognized as foreign or 
mismatched and subsequently repaired by the BER or mismatch repair (MMR) pathways creating mutations along the DNA [98-101]. PARP1/ARTD1 involvement has been implicated for BER and MMR, as studies where PARP1/ARTD1 was knockdown have resulted in alterations in repair of the DNA [102,103]. However, a study using a PCR based assay determined that PARP1/ARTD1 was not necessary for somatic hypermutation along with a number of other proteins involved in various DNA repair pathways [104]. Of note, these proteins were knockout independently and had no major effect on mutations accumulated in B cells, indicating that it is likely that not just one protein and one pathway but a crosstalk across various pathways is required to repair the programmed DNA damage. As discussed above for gene conversion, the frequency and success of increasing diversity is dependent upon the interplay between two pathways, PARP1/ARTD1- or Ku70-dependent, competing for access to repair the DNA. Aside from using single knockout mice, chemical inhibition of a family of proteins may provide more robust results, for example utilizing a pan-PARPs/ARTDs inhibitor to avoid compensatory activity of other PARPs/ARTDs in a PARP1/ARTD1 knockout mouse.

Strikingly, AID-deficient B cells also have decreased PARylation during class switch recombination [86], which implicates PARylation and PARPs/ARTDs in the recognition and regulation of the programmed DNA damage responses during these important recombination events. Unsurprisingly, AID dysregulation is thought to be largely associated with B cell lymphomas, a leading cause of cancer in the west $[98,105]$. Despite the various pathways used to sense and signal DNA damage, AID-induced DNA breaks can be atypically processed resulting in internal deletions and/or chromosomal translocations, similar to alternative NHEJ [106]. It is noteworthy that general PARP/ARTD inhibitors induced a four-fold increase in B cell chromosomal translocations [106]. PARP2/ARTD2-deficiency led to an even greater increase in translocation frequency, while PARP1/ARTD1 had no effect on translocation frequency. However, the use of a PARP/ARTD inhibitor in PARP2/ARTD2 deficient cells brought translocation levels to wild type, indicating that PARP1/ARTD1 may have role in regulating PARP2/ARTD2 activity [86].

Mature B cells further diversify their antigen receptors through class switch recombination (CSR) endowing antibodies with different effector functions. CSR is the process by which B cells can produce antibodies with similar variable regions, yet have different functions attributed to the heavy chain constant region [98]. During class switching, this constant region can be changed from the original IgH to any of the other isotypes: IgM to IgG, IgE, or IgA [101]. At a mechanistic level, AIDdependent deamination creates abasic sites that are cleaved into DSBs by endonucleases. These DSBs activate DNA damage response factors and repair through the NHEJ pathway [101]. The role of PARPs/ARTDs in class switching was first recognized approximately twenty years ago through the use of PARP inhibitors nicotinamide, 3-MB, and 1,5-diOH-Q. Murine B lymphoma cells treated with these inhibitors exhibited increased class switch recombination. Similarly, stimulated primary B lymphocytes also had recombination increases upon PARP/ARTD inhibition [107]. These early studies suggest that PARPs/ARTDs act as an inhibitor of class switching, possibly indicating its role as a negative regulator of the process. However, more recent studies have assayed PARP/ARTD activity in the CSR-proficient CH12 B lymphoma cell line and primary splenic B cells which showed PARylation of PARP1/ARTD1 when CSR was induced [86]. As previously mentioned, PARP1/ARTD1-deficient mice develop abnormal levels of basal immunoglobulins, having reduced levels of IgG2a and elevated $\operatorname{IgA}$ and $\operatorname{IgG} 2 \mathrm{~b}$, as well as altered levels of plasma and antigen-specific plasma cells [85] indicating 
that although these cells are technically mature there are deficits in their function. Inefficient repair of the programmed DNA damage may alter efficient class switch, keeping B cells from going through the normal progression of antibody production. Halting B cells in various stages of the class switch cycle could manifest itself in altered proportions of the antibody classes at the organism level; a similar conclusion was determined as the cause for having an altered repertoire of TCR $\alpha$ and reduced T cells in PARP2/ARTD2 deficient mice [90]. Examining the altered antibody responses in PARP1/ARTD1deficient mice, Ambrose and colleagues concluded that differences in class switch could be due to overall deficits in $\mathrm{T}$ cell dependent responses and germinal center function, but could not rule out other effects on survival of plasma cells [85]. One can speculate that in the absence of PARP1/ARTD1, inefficient repair of DSBs, induced during class switch, leads to apoptosis from DNA damage accumulation resulting in a lower total number of plasma cells and antigen specific plasma cells as was documented. In such studies it would be important to determine the difference between a lack of proliferation, increased cell death due to inadequate DNA repairs, or increased preference for specific sites of recombination. Understanding which of these explanations results in altered levels at the level of the whole animal will be important for guiding more nuanced mechanistic studies.

\section{Conclusion and Future Directions}

Intrinsic and extrinsic signals resulting in genotoxic stress and NF- $\kappa \mathrm{B}$ activation provide a robust cellular environment to study the far-reaching effects that PARylation and PARPs/ARTDs have on cellular processes and in determining cell fate. During lymphocyte development and differentiation, DNA damage repair must be tightly controlled to strike the prefect balance between increasing genetic variation and cellular functionality (via mutagenic DNA repair) versus maintaining genomic integrity and preventing oncogenic outcomes (error-free DNA repair). It is intriguing to contemplate that PARylation can regulate the extent of immunoglobulin gene conversion, somatic hypermutation, and class switching by directing the specific repair pathway employed to either have error free or mutagenic repair. PARPs/ARTDs and PAR are at the apex of DNA damage repair pathways due to their functions in recognizing, initiating signaling cascades, and acting as a docking platform for signaling complexes, yet they may also function as a bridge to the intrinsic NF- $\mathrm{BB}$ pathway, particularly in B cells.

The NF-kB complex has been shown to be required for all facets of B cell development from its original description as being required for Ig kappa light chain transcription in B cells [61] through its more recently attributed functions for B cell receptor editing [72]. Repairing programmed DNA damage is also fundamentally required for proper maturation of $\mathrm{B}$ cells and to generate a varied repertoire of antibodies. PAR metabolism has been characterized as necessary for initiating, amplifying, sustaining, and eventually turning off the DNA repair signaling pathways. It is noteworthy that the roles of NF- $\mathrm{KB}$ and PARylation in B cells were investigated in isolation in almost all previous studies. Strikingly, PAR synthesis was recently revealed to be crucial in orchestrating signaling cascades that lead to NF- $\mathrm{KB}$ activation in response to environmental DNA damage [78], which makes it likely that PARylation also platforms the programmed DNA damage-activated intrinsic NF- $\mathrm{B}$ signalosome in B cells. We therefore speculate that PAR/PARPs/ARTDs could provide a direct link and fill in the gaps in understanding both programmed DNA damage/repair and NF- $\mathrm{B}$ in $\mathrm{B}$ cell development, differentiation, and maturation (Figure 2). Earlier studies investigating the lymphocytes 
of PARP1/ARTD1, PARP2/ARTD2, or PARP3/ARTD3 single knockout mice have indicated the potential role of PARylation in B cell function. While these animals develop mature B cells, the levels of basal immunoglobulin are altered as previously discussed [85-87]. That said, further studies are needed to directly monitor the dynamic PAR levels, as well as their association with the intrinsic DNA damage lesions and NF- $\kappa \mathrm{B}$ activities, during $\mathrm{B}$ cell development, differentiation, and maturation. Moreover, PARP1/ARTD1, PARP2/ARTD2, and PARP3/ARTD3 are able to compensate each other's PAR-catalytic activity in either single knockout mice, which could mask the requirement of PARylation in B cell development and maturation.

Figure 2. Poly-ADP-ribosylation (PARylation) in B cell development, differentiation, and maturation. DNA damage repair and NF- $\mathrm{B}$ activity are known to be critical during $\mathrm{B}$ cell lymphogenesis (in black and bold). PARylation has emerged to orchestrate signaling cascades that lead to DNA repair, NF- $\mathrm{BB}$ activation, as well as their crosstalk, thus potentially contributing to the various $\mathrm{B}$ cell functions including development, differentiation, maturation, response and others (in grey).

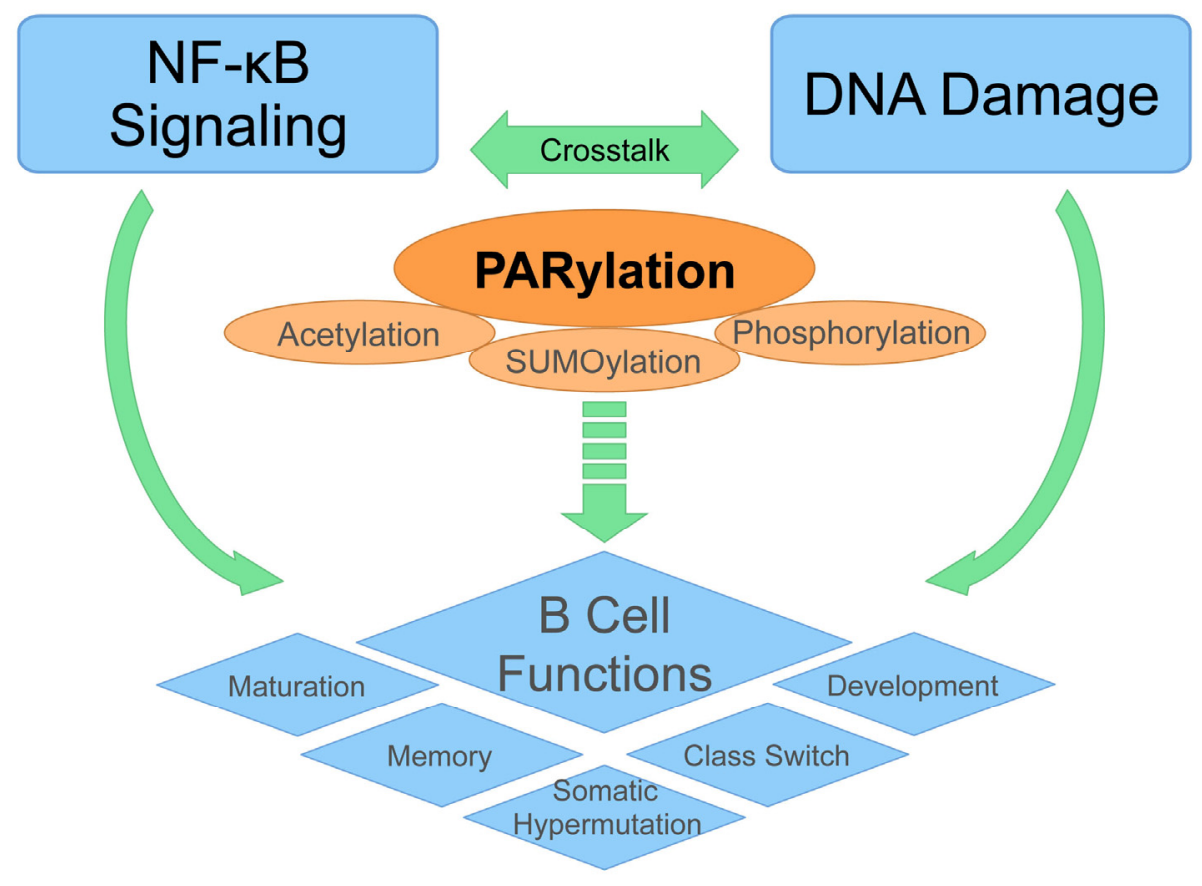

Future studies should also focus on understanding whether PARylation bridges the potential crosstalk between DNA damage repair and the $\mathrm{NF}-\mathrm{\kappa B}$ signaling pathway during $\mathrm{B}$ cell lymphomagenesis, given the promising therapeutic implications of NF- $\kappa B$ inhibitors in lymphoid malignancies [108]. While PARP1/ARTD1 and PAR provide a platform for the assembly of IKK $\gamma$, PIASy, and ATM in response to DNA damage [78] and mutations in IKK $\gamma$ specifically affect the initiation of the NF- $\kappa B$ DNA damage repair pathway [76,77], more needs to be done to determine how this ultimately affects B cell development, antibody maturation (somatic hypermutation, class switch, etc.), and if these defects will increase the likelihood of oncogenic events in affected B cells. Ultimately designing studies that are interdisciplinary in their approach will prove to be the most productive in understanding how PARylation, a PTM involved in regulating DNA damage responses, can influence 
adaptive immunity to infections and immune related cancers. Studies combining techniques and expertise from immunology, cell development, and DNA damage will provide the most valuable insight. There are numerous examples of the interconnectedness of cell signaling, demonstrating that it is no longer enough to study these pathways and proteins in an isolated field and the involvement of DNA damage repair proteins on the developing immune system and B cell diversity will likely be no different.

\section{Acknowledgements}

We apologize to those who also made important contributions to the issues discussed and could not be cited due to space limitations. A.H. is a Hopkins Sommer Scholar. Our work is supported by the National Cancer Institute and the American Cancer Society (to F.W.).

\section{Conflicts of Interest}

The authors declare no conflict of interest.

\section{References}

1. Hynes, N.E.; Ingham, P.W.; Lim, W.A.; Marshall, C.J.; Massague, J.; Pawson, T. Signalling change: Signal transduction through the decades. Nat. Rev. Mol. Cell Biol. 2013, 14, 393-398.

2. Schreiber, V.; Dantzer, F.; Ame, J.C.; de Murcia, G. Poly(ADP-ribose): Novel functions for an old molecule. Nat. Rev. Mol. Cell Biol. 2006, 7, 517-528.

3. Gibson, B.A.; Kraus, W.L. New insights into the molecular and cellular functions of poly(ADP-ribose) and PARPs. Nat. Rev. Mol. Cell Biol. 2012, 13, 411-424.

4. Krishnakumar, R.; Kraus, W.L. The PARP Side of the Nucleus: Molecular actions, physiological outcomes, and clinical targets. Mol. Cell 2010, 39, 8-24.

5. Ruf, A.; Mennissier de Murcia, J.; de Murcia, G.; Schulz, G.E. Structure of the catalytic fragment of poly(AD-ribose) polymerase from chicken. Proc. Natl. Acad. Sci. USA 1996, 93, 7481-7485.

6. Oliver, A.W.; Ame, J.C.; Roe, S.M.; Good, V.; de Murcia, G.; Pearl, L.H. Crystal structure of the catalytic fragment of murine poly(ADP-ribose) polymerase-2. Nucleic Acids Res. 2004, 32, 456-464.

7. Hottiger, M.O.; Hassa, P.O.; Luscher, B.; Schuler, H.; Koch-Nolte, F. Toward a unified nomenclature for mammalian ADP-ribosyltransferases. Trends Biochem. Sci. 2010, 35, 208-219.

8. de Murcia, J.M.; Niedergang, C.; Trucco, C.; Ricoul, M.; Dutrillaux, B.; Mark, M.; Oliver, F.J.; Masson, M.; Dierich, A.; LeMeur, M.; et al. Requirement of poly(ADP-ribose) polymerase in recovery from DNA damage in mice and in cells. Proc. Natl. Acad. Sci. USA 1997, 94, 7303-7307.

9. $\quad$ Masutani, M.; Nozaki, T.; Nishiyama, E.; Shimokawa, T.; Tachi, Y.; Suzuki, H.; Nakagama, H.; Wakabayashi, K.; Sugimura, T. Function of poly(ADP-ribose) polymerase in response to DNA damage: gene-disruption study in mice. Mol. Cell. Biochem. 1999, 193, 149-152.

10. Durkacz, B.W.; Omidiji, O.; Gray, D.A.; Shall, S. (ADP-ribose)n participates in DNA excision repair. Nature 1980, 283, 593-596.

11. Shall, S.; de Murcia, G. Poly(ADP-ribose) polymerase-1: What have we learned from the deficient mouse model? Mutat. Res. 2000, 460, 1-15. 
12. Zhang, Y.; Wang, J.; Ding, M.; Yu, Y. Site-specific characterization of the Asp- and Glu-ADPribosylated proteome. Nat. Methods 2013, 10, 981-984.

13. Jungmichel, S.; Rosenthal, F.; Altmeyer, M.; Lukas, J.; Hottiger, M.O.; Nielsen, M.L. Proteomewide identification of poly(ADP-Ribosyl)ation targets in different genotoxic stress responses. Mol. Cell 2013, 52, 272-285.

14. Loseva, O.; Jemth, A.S.; Bryant, H.E.; Schuler, H.; Lehtio, L.; Karlberg, T.; Helleday, T. PARP3 Is a Mono-ADP-ribosylase That Activates PARP-1 in the Absence of DNA. J. Biol. Chem. 2010, 285, 8054-8060.

15. Kraus, W.L. Transcriptional control by PARP-1: chromatin modulation, enhancer-binding, coregulation, and insulation. Curr. Opin. Cell Biol. 2008, 20, 294-302.

16. Potaman, V.N.; Shlyakhtenko, L.S.; Oussatcheva, E.A.; Lyubchenko, Y.L.; Soldatenkov, V.A. Specific Binding of Poly(ADP-ribose) Polymerase-1 to Cruciform Hairpins. J. Mol. Biol. 2005, 348, 609-615.

17. Cohen-Armon, M. PARP-1 activation in the ERK signaling pathway. Trends Pharmacol. Sci. 2007, 28, 556-560.

18. Krishnakumar, R.; Gamble, M.J.; Frizzell, K.M.; Berrocal, J.G.; Kininis, M.; Kraus, W.L. Reciprocal Binding of PARP-1 and Histone $\mathrm{H} 1$ at Promoters Specifies Transcriptional Outcomes. Science 2008, 319, 819-821.

19. Andrabi, S.A.; Kim, N.S.; Yu, S.-W.; Wang, H.; Koh, D.W.; Sasaki, M.; Klaus, J.A.; Otsuka, T.; Zhang, Z.; Koehler, R.C.; et al. Poly(ADP-ribose) (PAR) polymer is a death signal. Proc. Natl. Acad. Sci. USA 2006, 103, 18308-18313.

20. Koh, D.W.; Dawson, T.M.; Dawson, V.L. Poly-ADP-ribosylation in health and disease. Cell. Mol. Life Sci. 2005, 62, 760-768.

21. Yu, S.-W.; Andrabi, S.A.; Wang, H.; Kim, N.S.; Poirier, G.G.; Dawson, T.M.; Dawson, V.L. Apoptosis-inducing factor mediates poly(ADP-ribose) (PAR) polymer-induced cell death. Proc. Natl. Acad. Sci. USA 2006, 103, 18314-18319.

22. Rosenthal, F.; Feijs, K.L.; Frugier, E.; Bonalli, M.; Forst, A.H.; Imhof, R.; Winkler, H.C.; Fischer, D.; Caflisch, A.; Hassa, P.O.; et al. Macrodomain-containing proteins are new mono-ADP-ribosylhydrolases. Nat. Struct. Mol. Biol. 2013, 20, 502-507.

23. Jankevicius, G.; Hassler, M.; Golia, B.; Rybin, V.; Zacharias, M.; Timinszky, G.; Ladurner, A.G. A family of macrodomain proteins reverses cellular mono-ADP-ribosylation. Nat. Struct. Mol. Biol. 2013, 20, 508-514.

24. Min, W.; Wang, Z.Q. Poly (ADP-ribose) glycohydrolase (PARG) and its therapeutic potential. Front. Biosci. 2009, 14, 1619-1626.

25. Davidovic, L. Importance of Poly(ADP-Ribose) Glycohydrolase in the control of Poly(ADPRibose) metabolism. Exp. Cell Res. 2001, 268, 7-13.

26. Mortusewicz, O.; Fouquerel, E.; Ame, J.C.; Leonhardt, H.; Schreiber, V. PARG is recruited to DNA damage sites through poly(ADP-ribose)- and PCNA-dependent mechanisms. Nucleic Acids Res. 2011, 39, 5045-5056. 
27. Cortes, U.; Tong, W.M.; Coyle, D.L.; Meyer-Ficca, M.L.; Meyer, R.G.; Petrilli, V.; Herceg, Z.; Jacobson, E.L.; Jacobson, M.K.; Wang, Z.Q. Depletion of the 110-Kilodalton isoform of Poly(adp-ribose) glycohydrolase increases sensitivity to genotoxic and endotoxic stress in mice. Mol. Cell. Biol. 2004, 24, 7163-7178.

28. Gao, H.; Coyle, D.L.; Meyer-Ficca, M.L.; Meyer, R.G.; Jacobson, E.L.; Wang, Z.-Q.; Jacobson, M.K. Altered poly(ADP-ribose) metabolism impairs cellular responses to genotoxic stress in a hypomorphic mutant of poly(ADP-ribose) glycohydrolase. Exp. Cell Res. 2007, 313, 984-996.

29. Masutani, M.; Nakagama, H.; Sugimura, T. Poly(ADP-ribose) and carcinogenesis. Genes Chromosom. Cancer 2003, 38, 339-348.

30. Min, W.; Cortes, U.; Herceg, Z.; Tong, W.M.; Wang, Z.Q. Deletion of the nuclear isoform of poly(ADP-ribose) glycohydrolase (PARG) reveals its function in DNA repair, genomic stability and tumorigenesis. Carcinogenesis 2010, 31, 2058-2065.

31. Lukas, J.; Lukas, C.; Bartek, J. More than just a focus: The chromatin response to DNA damage and its role in genome integrity maintenance. Nat. Cell Biol. 2011, 13, 1161-1169.

32. Messner, S.; Altmeyer, M.; Zhao, H.; Pozivil, A.; Roschitzki, B.; Gehrig, P.; Rutishauser, D.; Huang, D.; Caflisch, A.; Hottiger, M.O. PARP1 ADP-ribosylates lysine residues of the core histone tails. Nucleic Acids Res. 2010, 38, 6350-6362.

33. Poirier, G.G.; de Murcia, G.; Jongstra-Bilen, J.; Niedergang, C.; Mandel, P. Poly(ADPribosyl)ation of polynucleosomes causes relaxation of chromatin structure. Proc. Natl. Acad. Sci. USA 1982, 79, 3423-3427.

34. Realini, C.A.; Althaus, F.R. Histone shuttling by poly(ADP-ribosylation). J. Biol. Chem. 1992, 267, 18858-18865.

35. Ahel, D.; Horejsi, Z.; Wiechens, N.; Polo, S.E.; Garcia-Wilson, E.; Ahel, I.; Flynn, H.; Skehel, M.; West, S.C.; Jackson, S.P.; et al. Poly(ADP-ribose)-Dependent Regulation of DNA Repair by the Chromatin Remodeling Enzyme ALC1. Science 2009, 325, 1240-1243.

36. Gottschalk, A.J.; Timinszky, G.; Kong, S.E.; Jin, J.; Cai, Y.; Swanson, S.K.; Washburn, M.P.; Florens, L.; Ladurner, A.G.; Conaway, J.W.; et al. Poly(ADP-ribosyl)ation directs recruitment and activation of an ATP-dependent chromatin remodeler. Proc. Natl. Acad. Sci. USA 2009, 106, 13770-13774.

37. Polo, S.E.; Kaidi, A.; Baskcomb, L.; Galanty, Y.; Jackson, S.P. Regulation of DNA-damage responses and cell-cycle progression by the chromatin remodelling factor CHD4. EMBO J. 2010, 29, 3130-3139.

38. Chou, D.M.; Adamson, B.; Dephoure, N.E.; Tan, X.; Nottke, A.C.; Hurov, K.E.; Gygi, S.P.; Colaiácovo, M.P.; Elledge, S.J. A chromatin localization screen reveals poly (ADP ribose)-regulated recruitment of the repressive polycomb and NuRD complexes to sites of DNA damage. Proc. Natl. Acad. Sci. USA 2010, 107, 18475-18480.

39. Okano, S.; Lan, L.; Caldecott, K.W.; Mori, T.; Yasui, A. Spatial and temporal cellular responses to single-strand breaks in human cells. Mol. Cell. Biol. 2003, 23, 3974-3981.

40. Mortusewicz, O.; Ame, J.C.; Schreiber, V.; Leonhardt, H. Feedback-regulated poly(ADPribosyl)ation by PARP-1 is required for rapid response to DNA damage in living cells. Nucleic Acids Res. 2007, 35, 7665-7675. 
41. Spagnolo, L.; Barbeau, J.; Curtin, N.J.; Morris, E.P.; Pearl, L.H. Visualization of a DNAPK/PARP1 complex. Nucleic Acids Res. 2012, 40, 4168-4177.

42. Haince, J.F.; Kozlov, S.; Dawson, V.L.; Dawson, T.M.; Hendzel, M.J.; Lavin, M.F.; Poirier, G.G. Ataxia telangiectasia mutated (ATM) signaling network is modulated by a novel poly(ADP-ribose)-dependent pathway in the early response to DNA-damaging agents. J. Biol. Chem. 2007, 282, 16441-16453.

43. McCool, K.W.; Miyamoto, S. DNA damage-dependent NF- $\kappa$ B activation: NEMO turns nuclear signaling inside out. Immunol. Rev. 2012, 246, 311-326.

44. Tramontano, F.; Malanga, M.; Quesada, P. Differential contribution of poly(ADPribose)polymerase-1 and -2 (PARP-1 and -2) to the poly(ADP-ribosyl)ation reaction in rat primary spermatocytes. Mol. Hum. Reprod. 2007, 13, 821-828.

45. Dantzer, F.; Mark, M.; Quenet, D.; Scherthan, H.; Huber, A.; Liebe, B.; Monaco, L.; Chicheportiche, A.; Sassone-Corsi, P.; de Murcia, G.; et al. Poly(ADP-ribose) polymerase-2 contributes to the fidelity of male meiosis I and spermiogenesis. Proc. Natl. Acad. Sci. USA 2006, 103, 14854-14859.

46. Meyer-Ficca, M.L.; Lonchar, J.D.; Ihara, M.; Meistrich, M.L.; Austin, C.A.; Meyer, R.G. Poly(ADP-Ribose) Polymerases PARP1 and PARP2 modulate topoisomerase II Beta (TOP2B) function during chromatin condensation in mouse spermiogenesis. Biol. Reprod. 2011, 84, 900-909.

47. Yang, F.; Baumann, C.; De La Fuente, R. Persistence of histone H2AX phosphorylation after meiotic chromosome synapsis and abnormal centromere cohesion in poly (ADP-ribose) polymerase (Parp-1) null oocytes. Develop. Biol. 2009, 331, 326-338.

48. Meyer-Ficca, M.L.; Lonchar, J.; Credidio, C.; Ihara, M.; Li, Y.; Wang, Z.Q.; Meyer, R.G. Disruption of Poly(ADP-Ribose) homeostasis affects spermiogenesis and sperm chromatin integrity in mice. Biol. Reprod. 2009, 81, 46-55.

49. Turlure, F.; Devroe, E.; Silver, P.A.; Engelman, A. Human cell proteins and human immunodeficiency virus DNA integration. Front. Biosci. 2004, 9, 3187-3208.

50. Yoder, K.E.; Bushman, F.D. Repair of gaps in retroviral DNA integration intermediates. J. Virol. 2000, 74, 11191-11200.

51. Gäken, J.A.; Tavassoli, M.; Gan, S.U.; Vallian, S.; Giddings, I.; Darling, D.C.; Galea-Lauri, J.; Thomas, M.G.; Abedi, H.; Schreiber, V.; et al. Efficient retroviral infection of mammalian cells is blocked by inhibition of poly(ADP-ribose) polymerase activity. J. Virol. 1996, 70, 3992-4000.

52. Kameoka, M.; Tanaka, Y.; Ota, K.; Itaya, A.; Yoshihara, K. Poly (ADP-ribose) polymerase is involved in PMA-induced activation of HIV-1 in U1 cells by modulating the LTR function. Biochem. Biophys. Res. Commun. 1999, 262, 285-289.

53. Kameoka, M.; Nukuzuma, S.; Itaya, A.; Tanaka, Y.; Ota, K.; Inada, Y.; Ikuta, K.; Yoshihara, K. Poly(ADP-ribose)polymerase-1 is required for integration of the human immunodeficiency virus type 1 genome near centromeric alphoid DNA in human and murine cells. Biochem. Biophys. Res. Commun. 2005, 334, 412-417.

54. Ha, H.C.; Juluri, K.; Zhou, Y.; Leung, S.; Hermankova, M.; Snyder, S.H. Poly(ADP-ribose) polymerase-1 is required for efficient HIV-1 integration. Proc. Natl. Acad. Sci. USA 2001, 98, 3364-3368. 
55. Baekelandt, V.; Claeys, A.; Cherepanov, P.; De Clercq, E.; De Strooper, B.; Nuttin, B.; Debyser, Z. DNA-Dependent protein kinase is not required for efficient lentivirus integration. J. Virol. 2000, 74, 11278-11285.

56. Siva, A.C.; Bushman, F. Poly(ADP-Ribose) Polymerase 1 is not strictly required for infection of murine cells by retroviruses. J. Virol. 2002, 76, 11904-11910.

57. Ariumi, Y.; Turelli, P.; Masutani, M.; Trono, D. DNA damage sensors ATM, ATR, DNA-PKcs, and PARP-1 are dispensable for human immunodeficiency virus Type 1 integration. J. Virol. 2005, 79, 2973-2978.

58. Jacelon, C.S.; Hanson, A. Older adults' participation in the development of smart environments: An integrated review of the literature. Geriatr. Nurs. 2013, 34, 116-121.

59. Boboila, C.; Alt, F.W.; Schwer, B. Classical and alternative end-joining pathways for repair of lymphocyte-specific and general DNA double-strand breaks. Adv. Immunol. 2012, 116, 1-49.

60. Puebla-Osorio, N.; Zhu, C. DNA damage and repair during lymphoid development: Antigen receptor diversity, Genomic integrity and lymphomagenesis. Immunol. Res. 2008, 41, 103-122.

61. Sen, R.; Baltimore, D. Multiple nuclear factors interact with the immunoglobulin enhancer sequences. Cell 1986, 46, 705-716.

62. Kaileh, M.; Sen, R. NF-kappaB function in B lymphocytes. Immunol. Res. 2012, 246, 254-271.

63. Gerondakis, S.; Siebenlist, U. Roles of the NF-kappaB pathway in lymphocyte development and function. Cold Spring Harb. Perspect. Biol. 2010, 2, a000182.

64. Vallabhapurapu, S.; Karin, M. Regulation and function of NF-kappaB transcription factors in the immune system. Annu. Rev. Immunol. 2009, 27, 693-733.

65. Hayden, M.S.; Ghosh, S. Shared principles in NF-kappaB signaling. Cell 2008, 132, 344-362.

66. Wan, F.; Anderson, D.E.; Barnitz, R.A.; Snow, A.; Bidere, N.; Zheng, L.; Hegde, V.; Lam, L.T.; Staudt, L.M.; Levens, D.; et al. Ribosomal protein S3: a KH domain subunit in NF-kappaB complexes that mediates selective gene regulation. Cell 2007, 131, 927-939.

67. Fu, K.; Sun, X.; Zheng, W.; Wier, E.M.; Hodgson, A.; Tran, D.Q.; Richard, S.; Wan, F. Sam68 modulates the promoter specificity of NF-kappaB and mediates expression of CD25 in activated T cells. Nat. Commun. 2013, 4, 1909.

68. Wan, F.; Lenardo, M.J. Specification of DNA binding activity of NF-kappaB proteins. Cold Spring Harb. Perspect. Biol. 2009, 1, a000067.

69. Wan, F.; Lenardo, M.J. The nuclear signaling of NF-kappaB: Current knowledge, New insights, and future perspectives. Cell Res. 2010, 20, 24-33.

70. Claudio, E.; Saret, S.; Wang, H.; Siebenlist, U. Cell-autonomous role for NF-kappa B in immature bone marrow B cells. J. Immunol. 2009, 182, 3406-3413.

71. Verkoczy, L.; Ait-Azzouzene, D.; Skog, P.; Martensson, A.; Lang, J.; Duong, B.; Nemazee, D. A role for nuclear factor kappa $\mathrm{B} /$ rel transcription factors in the regulation of the recombinase activator genes. Immunity 2005, 22, 519-531.

72. Cadera, E.J.; Wan, F.; Amin, R.H.; Nolla, H.; Lenardo, M.J.; Schlissel, M.S. NF-kappaB activity marks cells engaged in receptor editing. J. Exp. Med. 2009, 206, 1803-1816.

73. Allman, D.; Pillai, S. Peripheral B cell subsets. Curr. Opin. Immunol. 2008, 20, 149-157. 
74. Srinivasan, L.; Sasaki, Y.; Calado, D.P.; Zhang, B.; Paik, J.H.; DePinho, R.A.; Kutok, J.L.; Kearney, J.F.; Otipoby, K.L.; Rajewsky, K. PI3 kinase signals BCR-dependent mature B cell survival. Cell 2009, 139, 573-586.

75. Gerondakis, S.; Grumont, R.J.; Banerjee, A. Regulating B-cell activation and survival in response to TLR signals. Immunol. Cell Biol. 2007, 85, 471-475.

76. Jain, A.; Ma, C.A.; Liu, S.; Brown, M.; Cohen, J.; Strober, W. Specific missense mutations in NEMO result in hyper-IgM syndrome with hypohydrotic ectodermal dysplasia. Nat. Immunol. 2001, 2, 223-228.

77. Jain, A.; Ma, C.A.; Lopez-Granados, E.; Means, G.; Brady, W.; Orange, J.S.; Liu, S.; Holland, S.; Derry, J.M. Specific NEMO mutations impair CD40-mediated c-Rel activation and B cell terminal differentiation. J. Clin. Invest. 2004, 114, 1593-1602.

78. Stilmann, M.; Hinz, M.; Arslan, S.C.; Zimmer, A.; Schreiber, V.; Scheidereit, C. A Nuclear Poly(ADP-Ribose)-Dependent signalosome confers DNA damage-induced IкB kinase activation. Mol. Cell 2009, 36, 365-378.

79. Hassa, P.O.; Hottiger, M.O. A role of poly (ADP-ribose) polymerase in NF-kappaB transcriptional activation. Biol. Chem. 1999, 380, 953-959.

80. Hassa, P.O. The Enzymatic and DNA binding activity of PARP-1 are not required for NF-kappa B coactivator function. J. Biol. Chem. 2001, 276, 45588-45597.

81. Veuger, S.J.; Hunter, J.E.; Durkacz, B.W. Ionizing radiation-induced NF- $\kappa B$ activation requires PARP-1 function to confer radioresistance. Oncogene 2008, 28, 832-842.

82. Mabb, A.M.; Wuerzberger-Davis, S.M.; Miyamoto, S. PIASy mediates NEMO sumoylation and NF- $\kappa \mathrm{B}$ activation in response to genotoxic stress. Nat. Cell Biol. 2006, 8, 986-993.

83. Helmink, B.A.; Sleckman, B.P. The response to and repair of RAG-mediated DNA double-strand breaks. Annu. Rev. Immunol. 2012, 30, 175-202.

84. Schatz, D.G.; Ji, Y. Recombination centres and the orchestration of V(D)J recombination. Nat. Rev. Immunol. 2011, 11, 251-263.

85. Ambrose, H.E.; Willimott, S.; Beswick, R.W.; Dantzer, F.; de Murcia, J.M.; Yelamos, J.; Wagner, S.D. Poly(ADP-ribose) polymerase-1 (Parp-1)-deficient mice demonstrate abnormal antibody responses. Immunol. 2009, 127, 178-186.

86. Robert, I. Parp1 facilitates alternative NHEJ, whereas Parp2 suppresses IgH/c-myc translocations during immunoglobulin class switch recombination. J. Exp. Med. 2009, 206, 1047-1056.

87. Robert, I.; Karicheva, O.; Martin, B.R. S.; Schreiber, V.; Dantzer, F. Functional aspects of PARylation in induced and programmed DNA repair processes: Preserving genome integrity and modulating physiological events. Mol. Aspects Med. 2013, 34, 1-15.

88. Yelamos, J.; Schreiber, V.; Dantzer, F. Toward specific functions of poly(ADP-ribose) polymerase-2. Trends Mol. Med. 2008, 14, 169-178.

89. Nicolas, L.; Martinez, C.; Baro, C.; Rodriguez, M.; Baroja-Mazo, A.; Sole, F.; Flores, J.M.; Ampurdanes, C.; Dantzer, F.; Martin-Caballero, J.; et al. Loss of poly(ADP-ribose) polymerase2 leads to rapid development of spontaneous T-cell lymphomas in p53-deficient mice. Oncogene 2010, 29, 2877-2883. 
90. Yelamos, J.; Monreal, Y.; Saenz, L.; Aguado, E.; Schreiber, V.; Mota, R.; Fuente, T.; Minguela, A.; Parrilla, P.; de Murcia, G.; et al. PARP-2 deficiency affects the survival of CD4+CD8+ double-positive thymocytes. EMBO J. 2006, 25, 4350-4360.

91. Arakawa, H. Requirement of the Activation-Induced Deaminase (AID) gene for Immunoglobulin gene conversion. Science 2002, 295, 1301-1306.

92. Tang, E.S.; Martin, A. Immunoglobulin gene conversion: Synthesizing antibody diversification and DNA repair. DNA Repair 2007, 6, 1557-1571.

93. Paddock, M.N.; Bauman, A.T.; Higdon, R.; Kolker, E.; Takeda, S.; Scharenberg, A.M. Competition between PARP-1 and Ku70 control the decision between high-fidelity and mutagenic DNA repair. DNA Repair 2011, 10, 338-343.

94. Paddock, M.N.; Buelow, B.D.; Takeda, S.; Scharenberg, A.M. The BRCT domain of PARP-1 is required for immunoglobulin gene conversion. PLoS Biol. 2010, 8, e1000428.

95. Wang, M.; Wu, W.; Wu, W.; Rosidi, B.; Zhang, L.; Wang, H.; Iliakis, G. PARP-1 and Ku compete for repair of DNA double strand breaks by distinct NHEJ pathways. Nucleic Acids Res. 2006, 34, 6170-6182.

96. Mansour, W.Y.; Borgmann, K.; Petersen, C.; Dikomey, E.; Dahm-Daphi, J. The absence of Ku but not defects in classical non-homologous end-joining is required to trigger PARP1-dependent end-joining. DNA Repair 2013, 12, 1134-1142.

97. Ma, J.L.; Kim, E.M.; Haber, J.E.; Lee, S.E. Yeast Mre11 and Rad1 proteins define a $\mathrm{Ku}$-independent mechanism to repair double-strand breaks lacking overlapping end sequences. Mol. Cell. Biol. 2003, 23, 8820-8828.

98. Nussenzweig, A.; Nussenzweig, M.C. Origin of chromosomal translocations in lymphoid cancer. Cell 2010, 141, 27-38.

99. Pavri, R.; Nussenzweig, M.C. AID Targeting in Antibody Diversity. Adv. Immunol. 2011, 110, $1-26$.

100. Di Noia, J.M.; Neuberger, M.S. Molecular mechanisms of antibody somatic hypermutation. Annu. Rev. Biochem. 2007, 76, 1-22.

101. Xu, Z.; Zan, H.; Pone, E.J.; Mai, T.; Casali, P. Immunoglobulin class-switch DNA recombination: induction, targeting and beyond. Nat. Rev. Immunol. 2012, 12, 517-531.

102. Dantzer, F.; de La Rubia, G.; Menissier-De Murcia, J.; Hostomsky, Z.; de Murcia, G.; Schreiber, V. Base excision repair is impaired in mammalian cells lacking Poly(ADP-ribose) polymerase-1. Biochemistry 2000, 39, 7559-7569.

103. Liu, Y.; Kadyrov, F.A.; Modrich, P. PARP-1 enhances the mismatch-dependence of 5'-directed excision in human mismatch repair in vitro. DNA Repair 2011, 10, 1145-1153.

104. Jacobs, H.; Fukita, Y.; van der Horst, G.T.; de Boer, J.; Weeda, G.; Essers, J.; de Wind, N.; Engelward, B.P.; Samson, L.; Verbeek, S.; et al. Hypermutation of immunoglobulin genes in memory B cells of DNA repair-deficient mice. J. Exp. Med. 1998, 187, 1735-1743.

105. Küppers, R. Mechanisms of B-cell lymphoma pathogenesis. Nat. Rev. Cancer 2005, 5, 251-262.

106. Robbiani, D.F.; Nussenzweig, M.C. Chromosome translocation, B cell lymphoma, and activation-induced cytidine deaminase. Annu. Rev. Pathol. Mech. Dis. 2013, 8, 79-103.

107. Shockett, P.; Stavnezer, J. Inhibitors of poly(ADP-ribose) polymerase increase antibody class switching. J. Immunol. 1993, 151, 6962-6976. 
108. Lim, K.H.; Yang, Y.; Staudt, L.M. Pathogenetic importance and therapeutic implications of NF-kappaB in lymphoid malignancies. Immunol. Rev. 2012, 246, 359-378.

(C) 2014 by the authors; licensee MDPI, Basel, Switzerland. This article is an open access article distributed under the terms and conditions of the Creative Commons Attribution license (http://creativecommons.org/licenses/by/3.0/). 